\title{
Glial-Guided Granule Neuron Migration in vitro: A High-Resolution Time-Lapse Video Microscopic Study
}

\author{
James C. Edmondson and Mary E. Hatten \\ Department of Pharmacology, New York University Medical Center, New York, New York 10016
}

\begin{abstract}
To study neuronal migration, migrating granule neurons in microcultures prepared from early postnatal cerebellum have been analyzed with time-lapse, video-enhanced differential interference contrast microscopy. The morphology of migrating neurons resembles the elongated forms of $\mathrm{mi}$ grating neurons described both in vivo and in vitro (Rakic, 1971; Hatten et al., 1984). The neuron closely apposes its soma along the glial fiber and extends a thickened leading process in the direction of migration. This leading tip is highly motile, with several filopodial extensions. Intracellular vesicular structures extend from the nucleus into the leading process of migrating neurons in vitro.

Quantitation of the motions of migrating neurons revealed a saltatory pattern of advance along the glial fiber. Periods of cell soma movement at the rate of $56 \pm 26 \mu \mathrm{m} / \mathrm{hr}$ along the glial fiber are punctuated by periods during which the cell soma slows to a complete stop. The overall rate of $\mathrm{mi}$ gration is $33 \pm 20 \mu \mathrm{m} / \mathrm{hr}$. The growing tip of the leading process rapidly extends and retracts, resulting in a net advance along the glial fiber. However, the periods of the extension and retraction of the leading process growing tip are not synchronized with the motions of the cell soma.
\end{abstract}

One of the best studied aspects of the development of the cerebellar cortex is the inward migration of young granule neurons along Bergmann fibers. The bipolar shape of the migrating granule neuron was first described from Golgi-impregnation studies by Ramón y Cajal (1911), who noted that the cell soma is preceded in its straight, inward migration by a thickened protoplasmic arm, called the leading process, while it leaves behind a branched axon. The cytology and cell-cell interactions of migrating granule neurons were analyzed in detail by Rakic (1971), who observed contacts between the leading process and one or more Bergmann astroglial fibers, which span the molecular layer and are oriented radially within the cerebellar cortex. Rakic proposed that the migration of granule neurons was guided by the Bergmann fibers. Defects in the interaction of migrating neurons with Bergmann fibers lead to a failure of granule neuron

Received Oct. 13, 1986; revised Dec. 8, 1986; accepted Jan. 8, 1987.

We gratefully acknowledge the extensive advice of Dr. Carol A. Mason and thank our colleagues Drs. David Colman, William Gregory, Michael Shelanski, and Ekkhart Trenkner for their criticisms. The photographic plates were prepared by Susan Babunovic and Peter Pierce. This research was submitted in partial fulfillment of the requirements for the Ph.D. degree. Supported by NIH Grant NS 15429 to M.E.H. J.C.E. is an NIH Medical Scientist Training Program fellow.

Correspondence should be addressed to Mary E. Hatten, Ph.D., Department of Pharmacology, New York University Medical Center, 550 First Avenue, New York, NY 10016.

Copyright (c) 1987 Society for Neuroscience $0270-6474 / 87 / 061928-07 \$ 02.00 / 0$ migration in the neurological mutant weaver mouse (Rakic and Sidman, 1973; Sotelo and Changeaux, 1974; Hatten et al., 1986).

$\Lambda$ dynamic view of granule neuron migration has bccomc available with several in vitro model systems. Trenkner and Sidman (1977) plated dissociated cerebellar cells in microwells on a non-adhesive substratum to promote the formation of cellular reaggregates. Thick cables consisting of numerous neuronal and astroglial fibers formed between the reaggregates within 24-48 hr, and granule neuron migration occurred along them.

A dissociated monolayer microculture system has been developed in our laboratory in which the migration of individual granule neurons along processes of Bergmann-like astroglial cells has been studied using phase-contrast optics (Hatten et al., 1984). Neuronal migration was seen only on astroglial cells that had highly elongated processes, and general aspects of granule cell movement along the glial process-apposition of the neuronal cell soma and extension of a thick leading process along the glial arm-resembled the features of migrating granule neurons in vivo described by Rakic (1971). Although this study yiclded much general information, including a direct demonstration of glial-guided neuronal migration, many details could not be resolved. Most notably, the behavior of the neuronal leading process and the arrangment of intracellular organelles could not be seen.

In the present study, migrating granule neurons in vitro were observed using the video-enhanced differential interference contrast technique of Allen et al. (1981), which allows high-contrast resolution of structures as small as $0.2 \mu \mathrm{m}$ in diameter in living tissue. We report that in vitro migrating granule neurons differ significantly in their cytology from nonmigrating granule neurons and that the motions of the leading process resemble aspects of the motions of both neuronal growth cones and migrating fibroblasts.

\section{Materials and Methods}

Cerebellar microcultures. Two different methods were used to prepare cultures for microscopic observation of migration. First, primary cultures were prepared from 5- to 7-d-old C57BL/6J mice as described previously (Hatten and Liem, 1981) and were observed after 48-72 hr. The predominant astroglial form under these conditions was the stellate astrocyte, a form that anchors neurons rather than supports their migration (Hatten et al., 1984). A small fraction of astroglia in these cultures expressed elongated, Bergmann-like forms. Migration of a few neurons was observed on Bergmann-like fibers after 48-72 hr; however, the percentage of granule neurons that migrated was relatively low in these cultures (less than $0.1 \%$, data not shown), which prompted a search for culture conditions that would yield a higher fraction of migrating cells.

In the second set of culture conditions, purified populations of astroglia and granule neurons of greater than $98 \%$ purity were prepared by a cell-separation technique described previously (Hatten, 1985). Sub- 

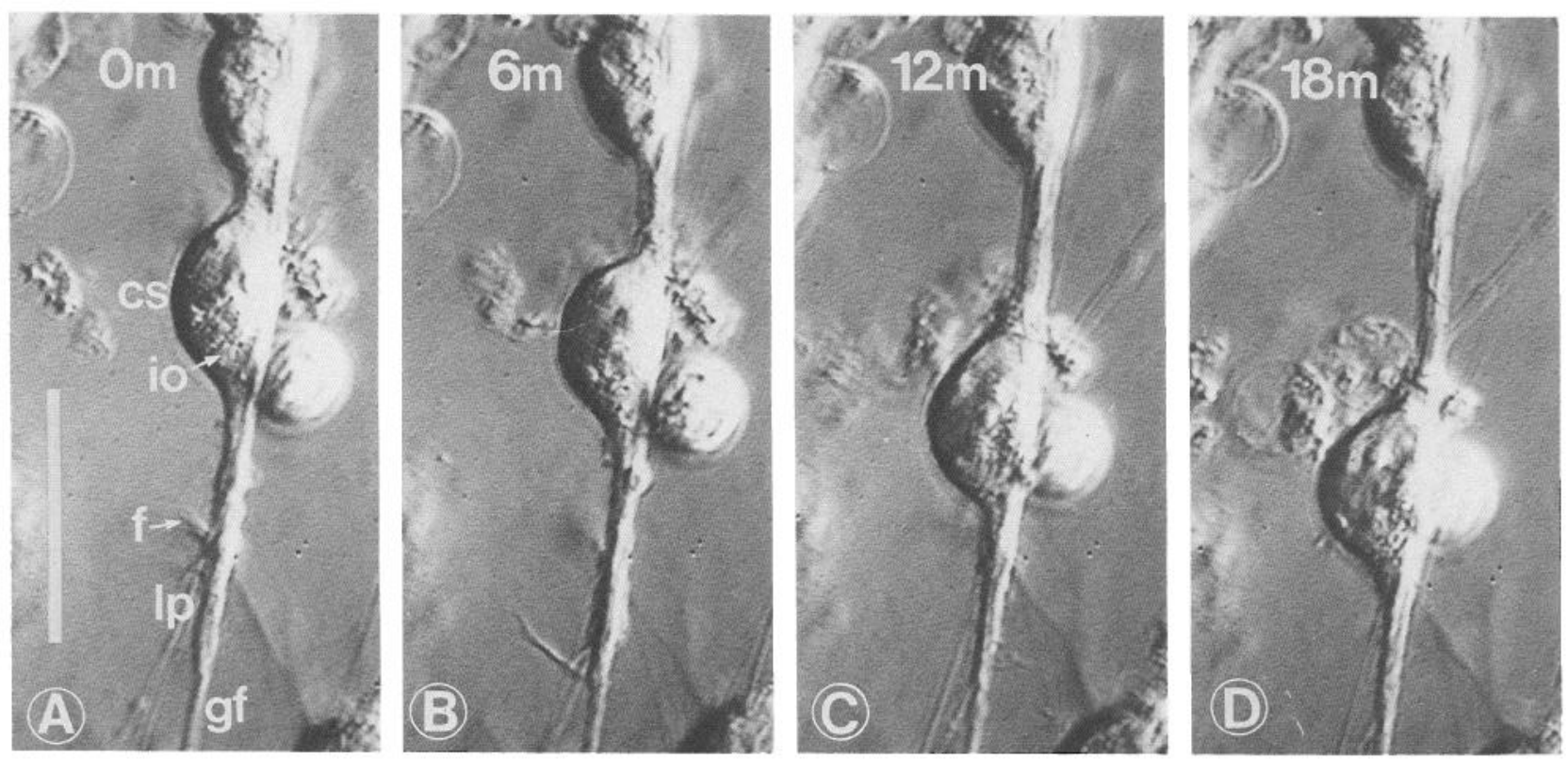

Figure 1. Cytology of a migrating granule neuron. $c s$, Cell soma; $i$, intracellular organelles; $l p$, leading process; $f$, filopodia; $g f$, glial fiber. Time interval (in min) indicated on photographs. Bar, $20 \mu \mathrm{m}$.

confluent cultures of purified astroglia were grown in isolation for a day, seeded on the second day with purified granule neurons at the ratio of 5-10 neurons per glial cell, cultured an additional day, and observed. Under these conditions, elongated glial forms that supported the migration of granule neurons were present within $24 \mathrm{hr}$ of the addition of granule neurons, and numerous migrating neurons were seen.

Preparation of culture dishes for microscopic observation. An 8-mmdiameter hole was punched in the center of petri dishes (Falcon 1006), and an $18 \mathrm{~mm} \# 1$ coverslip was attached with a 3:1 mixture of paraffin: vaseline to the bottom of the dish to cover the hole. Culture medium containing cells was added to form a convex meniscus, and cultures were placed in a humidified, $5 \% \mathrm{CO}_{2}, 35.5^{\circ} \mathrm{C}$ incubator (Napco). When cells were to be observed, cultures were removed from the incubator and a $25 \mathrm{~mm} \# 1$ coverslip was placed immediately on the meniscus of culture medium and sealed with silicon vacuum grease (Dow Corning). The sealed chamber was placed on the stage of a Zeiss IM microscope enclosed in a clear plastic bag. The specimen, objectives, and condenser were heated to $35.5^{\circ} \mathrm{C}$ by a thermostatically controlled Leitz stage heater.

Video-enhanced differential interference contrast microscopy. The Zeiss IM microscope was equipped with Nomarski differential interference contrast optics. Light from a $50 \mathrm{~W}$ mercury arc lamp was passed through a $546 \mathrm{~nm}$ (green) interference filter and adjusted for Köhler illumination. The image from a $63 \times / 1.4$ NA planapochromat oil-immersion objective was projected through a $20 \times$ eyepiece onto a Hamamatsu C196501 video camera. To enhance contrast according to the method of Allen et al. (1981), the video gain was increased as an offset voltage was applied; this increases the range of brightness levels between black and white and decreases the background gray level, respectively.

Immunocytochemistry. After recording the motions of migrating cells, the location of the observed cell was marked on the coverslip with a Leitz diamond-tipped object marker and the culture was fixed with $4 \%$ paraformaldehyde by flooding the petri dish and lifting the top coverslip. After fixation for $30 \mathrm{~min}$, cultures were stained immunocytochemically to localize the glial filament protein using a primary antibody obtained from our colleague, Dr. Ronald Liem, as described previously (Hatten and Liem, 1981). The stained culture was used to locate and identify the process that guided migration.

Time-lapse video recording and photography. The enhanced video signal was processed through a time-date generator(MSI Video Systems, Inc.) and was recorded either onto an NEC VC-9507 time-lapse videocassette recorder set at 1:90 time compression or at the rate of $1 \mathrm{frame} / 4$ $\mathrm{sec}$ onto a Panasonic optical memory disc recorder. Photographs were taken directly off the video monitor (Sony PVM-122) onto Kodak Technical Pan Film with a Nikon $35 \mathrm{~mm}$ camera set at ASA 80 and $1 / 4 \mathrm{sec}$ exposure time.

\section{Results}

In this study, more than 200 cultures were observed for periods of time ranging from $30 \mathrm{~min}$ to $18 \mathrm{hr}$. The distinction between migrating and nonmigrating (stationary) neurons was made on the basis of cellular movement. Only those neurons that advanced one cell diameter or more along the glial fiber during the period of observation were included in the migrating neuron category. By this criterion, more than 300 stationary and more than 40 migrating cells were observed with either phase-contrast or differential interference contrast microscopy. Of these, 14 migrating neurons were recorded with the high-resolution video microscope system and were analyzed in detail for presentation here.

\section{Cytological features of migrating and stationary neurons}

Migrating neurons. To locate migrating granule neurons, we looked for neuronal cell somata that were apposed to and flattened against Bergmann-like glial fibers, a posture described by Rakic (1971) for migrating neurons in vivo and by Hatten et al. (1984) for neurons in vitro (Fig. 1). Migrating granule neuron cell somata were $12 \pm 1.5 \mu \mathrm{m}$ long and $6 \pm 1 \mu \mathrm{m}$ wide. Extending from the cell soma in the direction of migration was a leading process $19 \pm 3.5 \mu \mathrm{m}$ long that tapered in width from about 2 $\mu \mathrm{m}$ at its origin on the cell soma to less than $1 \mu \mathrm{m}$ at the tip (Table 1). The leading process was uniformly and closely apposed to the glial fiber. Filopodia $1-5 \mu \mathrm{m}$ in length and $0.5-1$ $\mu \mathrm{m}$ in width originated on the leading process, on the soma, and on the trailing process of the migrating granule neuron. The direction of filopodial extension varied, as some filopodia extended up into the culture medium, while others adhered to the glial fiber. Filopodia of glial origin as long as $10 \mu \mathrm{m}$ were also observed. Intracellular organelles within migrating granule neurons extended into the leading process. Behind the migrating neuron, a thin trailing process that did not always contact the glial fiber was seen (Fig. 2).

Stationary neurons. To test whether stationary neurons ex- 

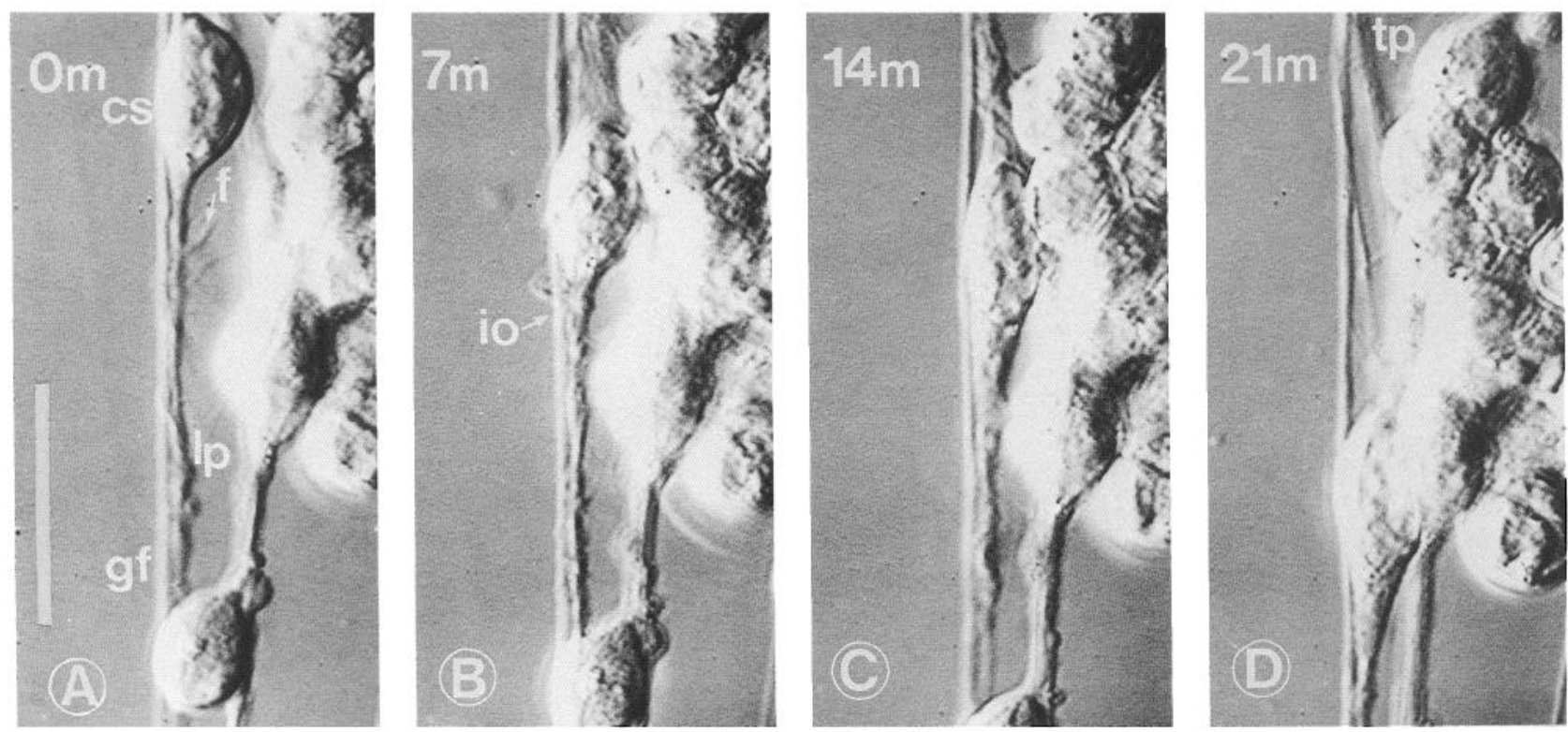

Figure 2. Behavior of the leading and trailing processes of a migrating granule neuron. Note that the leading process is always apposed to the glial fiber, whereas the trailing process extends away from the glial fiber. $c s$, Cell soma of migrating neuron; $l p$, leading process; $t p$, trailing process; $i o$, intracellular organelles; $f$, filopodia; $g f$, glial fiber. Time interval (in min) indicated on photographs. Bar, $20 \mu \mathrm{m}$.

hibited a nonrandom arrangement of intracellular organelles, the cell somata of 100 stationary neurons were divided into quadrants with each axis $45^{\circ}$ away from the associated glial fiber. Scoring cells for the quadrant that contained the intracellular vesicles indicated that there was no particular orientation of the vesicles relative to the axis of the glial process (Table 2). In addition, time-lapse video microscopy showed that in about $5 \%$ of the stationary neurons the entire nucleus-vesicle complex rotated completely within the rounded cell soma during a 10 min time period.

\section{Dynamic aspects of glial-guided granule neuron migration}

A summary of the dimensions and movements of 14 migrating neurons is presented in Table 1 . The average rate of migration for granule neurons was $33 \pm 20 \mu \mathrm{m} / \mathrm{hr}$. During periods of migration, advance of the cell soma was saltatory. To analyze the pattern of advance, we measured the position of both the cell soma and the tip of the leading process on the glial fiber at regular intervals. The measurements of the position of the cell soma showed that, while neurons migrated, periods of advance

Table 1. Summary of dimensions and movements of 14 migrating granule neurons

\begin{tabular}{lc} 
Variable & $\begin{array}{l}\text { Observed } \\
\text { value }\end{array}$ \\
\hline Soma length $(\mu \mathrm{m})$ & $12 \pm 1.5$ \\
Soma width $(\mu \mathrm{m})$ & $6 \pm 1$ \\
Leading process length $(\mu \mathrm{m})$ & $19 \pm 3.5$ \\
Average speed $(\mu \mathrm{m} / \mathrm{hr})$ & $33 \pm 20$ \\
$\begin{array}{l}\text { Average speed of moving cell soma }(\mu \mathrm{m} / \mathrm{hr}) \\
\text { Average duration of soma movement }(\mathrm{min})\end{array}$ & $56 \pm 26$ \\
$\begin{array}{l}\text { Average duration of soma pauses }(\mathrm{min}) \\
\text { Average distance traveled during cell soma movement } \\
\quad(\mu \mathrm{m})\end{array}$ & $4.8 \pm 1.6$ \\
& \\
\hline
\end{tabular}

of the cell soma averaging $4.8 \pm 1.6 \mathrm{~min}$ in duration were punctuated by periods of rest averaging $4.3 \pm 2.1 \mathrm{~min}$ in duration (Fig. $3 A$ ). The shift between movement and rest phases occurred over the course of 1-2 min.

At the onset of the cell soma movement cycle, the soma rapidly accelerated from rest to an average rate of $56 \pm 26 \mu \mathrm{m} /$ $\mathrm{hr}$. Depending on the duration of the phase of movement and the rate of migration, the cell soma advanced an average of $4 \pm$ $2 \mu \mathrm{m}$ during each phase of movement. At the end of each movement phase, the cell soma decelerated rapidly, often to a complete stop, to begin the rest phase.

By measuring the position of the growing tip of the leading process at very brief intervals, we found that it extended and retracted rapidly in an irregular pattern (Fig. $3 B$ ). On average, more time was spent extending than retracting, which led to gradual advance of the growing tip at the same overall rate as the cell soma from which it arose. The motions of the growing tip were not synchronized with those of the cell soma.

In several cases in which cultures were observed for longer time periods, neurons migrated for $30-120 \mathrm{~min}$, then paused for 1 or $2 \mathrm{hr}$ and then resumed their migration in the same direction. Thus, at any one time, only a subset of neurons with the cytological features of migrating cells actually migrated.

In some cases, the cessation of migration was accompanied by retraction of the leading process. Such a change often occurred as a migrating neuron reached the end of its glial guide fiber (Fig. 4). The converse transformation, from a rounded stationary to a flattened migrating shape, was also seen in the sample we studied (not shown).

A prominent feature of the leading process was the activity of its filopodia. Growth from the base of most filopodia was in the direction of migration or at a small angle away from it. The distal end of the filopodia often rotated backwards, falling against the body of the leading process, where resorption occurred (Fig. 5). Although filopodia were highly active on the leading process, they did not appear to pull the process forward. 

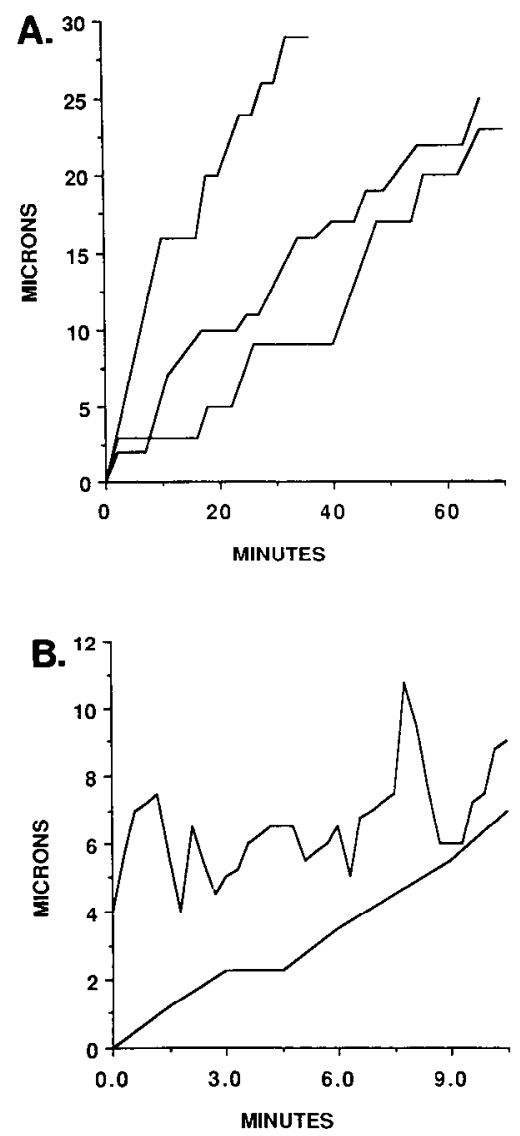

Figure 3. A, Graph showing the distance traveled by 3 separate granule neurons versus time. The position of each neuron was recorded at $2-\mathrm{min}$ intervals. Data points at the start of an upward-sloping segment represent the beginning of periods of movement at an average rate of $56 \pm$ $26 \mu \mathrm{m} / \mathrm{hr}$ along the glial fiber. Data points at the beginning of horizontal segments represent the beginning of periods of rest of the cell soma. The overall rate of migration for all cells was $33 \pm 20 \mu \mathrm{m} / \mathrm{hr}$. $B$, Graph showing the behavior of the cell soma and the growing tip of the leading process of a representative migrating neuron over a $10 \mathrm{~min}$ time span. The position of both the cell soma and the most distal extension of the leading process growing tip was recorded at $18 \mathrm{sec}$ intervals. The position of the growing tip (top line) was plotted at $18 \mathrm{sec}$ intervals. The position of the cell soma (bottom line) was plotted at $1 \frac{1 / 2}{2}$ min intervals, for clarity. The 2 lines were plotted on the same time scale, while the origin of the top line was arbitrarily placed $4 \mu \mathrm{m}$ above that for the bottom line for clarity. Note that the growing tip extends and retracts rapidly and that its motions are out of synchrony with the movements of the cell soma. These measurements were made over $10 \mathrm{~min}$, during which time the cell soma was advancing. The growing tip continued to extend and retract rapidly when the cell soma rested (not shown).

\section{Interaction of a migrating granule neuron with a second glial fiber in its path}

In the intact cerebellum, migrating granule neurons often contact more than one Bergmann fiber, particularly at the beginning of their migration (Rakic, 1971). Although it was not possible to align individual Bergmann fibers in the microcultures in order to observe this event, such an arrangement did arise fortuitously in one of the 14 cases of migration we observed. In this sequence, the migrating neuron switched its leading process from one glial fiber to another (Fig. 6).

The second glial fiber crossed the path of the migrating neuron at a narrow angle. The initial contact of the second glial fiber by the neuron was made by leading process, which bound rapidly
Table 2. Tabulation of the orientation relative to the glial process of intracellular organelles within 100 nonmigrating neurons

\begin{tabular}{ll} 
Orientation & Number \\
\hline Against the glial process & 27 \\
Towards a neurite in either direction & 49 \\
Away from the glial process & 24
\end{tabular}

$\overline{\text { A cross was superimposed on } 100 \text { nonmigrating neurons, with the center over the }}$ nucleus. Each axis was oriented $45^{\circ}$ away from the glial process contacted by the neuron. The orientation of the intracellular organelles relative to the glial process was scored by noting which quadrant contained the organelles (the totals for the 2 quadrants neither against nor away from the glial process being summed).

to the second fiber while retaining its apposition to the first. At the point of intersection, the neuron paused, after which the leading process switched to the second fiber and the cell continued its migration.

\section{Interaction of a migrating cell with a stationary cell on the same fiber}

In 2 of the 14 cases we studied, a migrating granule neuron approached and passed a stationary neuron on the same glial fiber (Fig. 7). As the migrating neuron approached the rounded, stationary ncuron, filopodia on the leading process contacted the stationary neuron. The migrating neuron slowed its migration and positioned its leading process beneath the stationary neuron, lifting the stationary neuron off of the glial fiber. After the cell soma of the migrating neuron passed beneath the stationary neuron, a close apposition was again seen between the stationary neuron and the glial fiber, and the migrating neuron resumed its usual rate of migration.

\section{Discussion}

In this study, the cytology and behavior of living, migrating cerebellar granule neurons were analyzed. The cytology of migrating neurons in vitro resembled that of migrating granule neurons in vivo. In the time-lapse experiments, granule neurons extended the leading process by rapid extension and retraction of the growing tip, while they moved the cell soma in a more regular saltatory pattern. Although the growing tip of the leading process in vitro resembled the tapered point seen in vivo, a feature found in vitro, but not in vivo, was the presence of filopodia. This difference may have been due to the interaction of granule neurons or the Bergmann-like astroglia with the culture substrate, to the lack of a surrounding neuropil in vitro, or to unknown factors. Alternatively, the short filopodia seen on migrating neurons in vitro might not be visible in vivo because of their sensitivity to fixation. The finding that filopodia on the growing tip of the migrating neuron did not appear to pull the process forward is consistent with recent views of neurite extension (Marsh and Letourneau, 1984; J. M. Aletta and L. A. Greene, unpublished observations). The function of these filopodia is unknown at present.

The video microscope system allowed observation of intracellular organelles in migrating granule neurons. It was of interest that in stationary neurons, the nucleus-vesicle complex was not strictly oriented relative to surrounding glial fibers. In migrating neurons, in contrast, intracellular vesicles invariably extended from the nuclear indentation into the leading process, suggesting that migrating neurons are highly polarized cells. It was not possible to determine the identity of the organelles due to resolution limits in the light microscope, although a number 

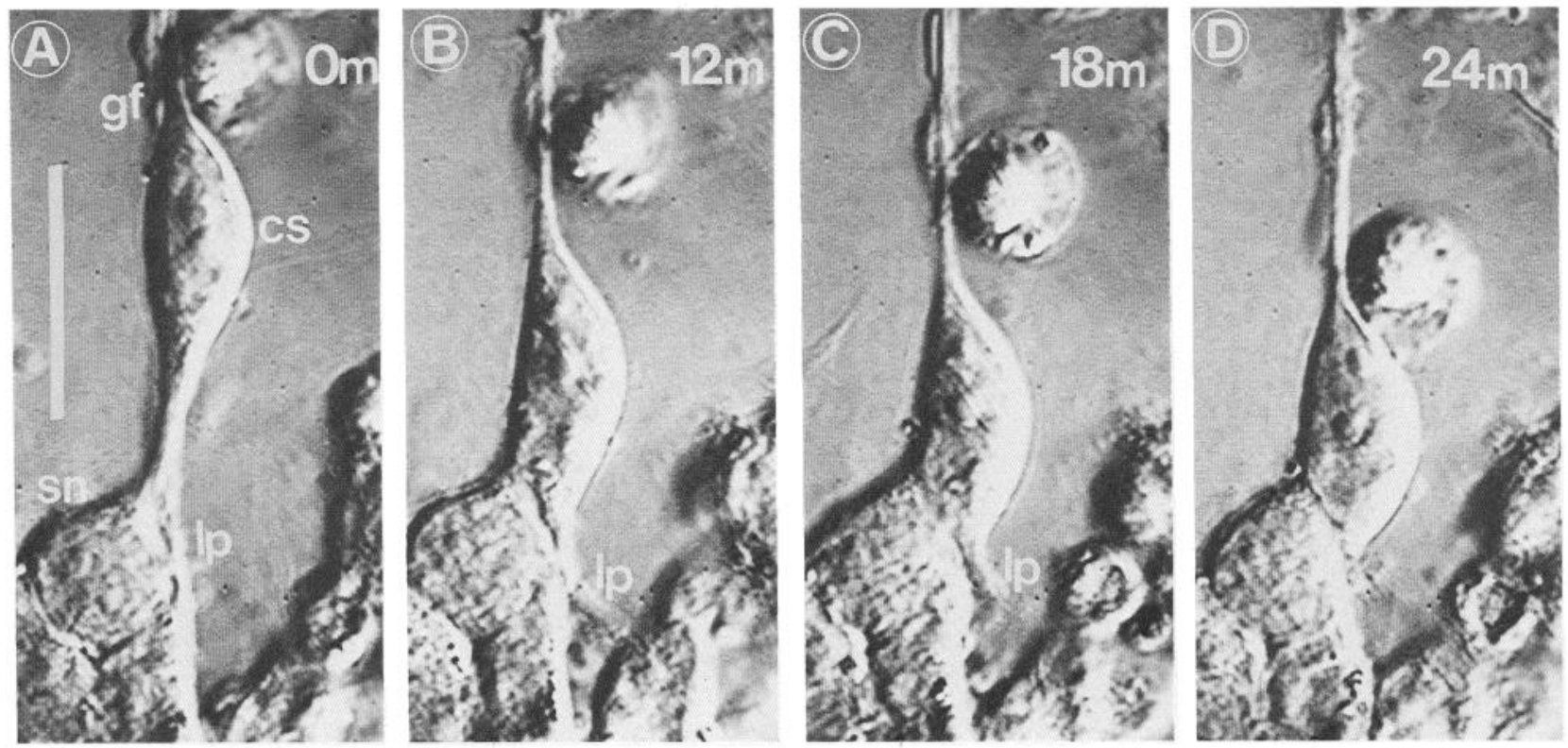

Figure 4. Release of the leading process at the end of a glial fiber. $c s$, Cell soma; $l p$, leading process; $s n$, stationary neuron; $g f$, glial fiber. $A$, Neuron migrates towards end of glial fiber. $B-D$, Neuron retracts the leading process at the end of the glial fiber. Time interval (in min) indicated on photographs. Bar, $20 \mu \mathrm{m}$.

of likely candidates have been seen in electron micrographs of video-identified migrating neurons, including mitochondria, coated vesicles, and Golgi apparatus (Gregory et al., 1986).

The morphology of the Bergmann-like glial fibers in the microculture system has been discussed in detail by Hatten et al. (1984). The present findings augment the prior descriptions with observations on the surface ruffling of living Bergmann-like glial fibers. The Bergmann-like glial processes that supported migration in vitro lacked the spines seen on Bergmann fibers in vivo (Rakic, 1971). That glial spines may not be needed for support of migration is consistent with the observation in vivo that these spines are absent at the site where the granule neuron leading process contacts the Bergmann fiber. In the present study, filopodia not resembling the spines seen in vivo sometimes extended from the Bergmann-like fibers.

The "start-stop" pattern of movement of the neuronal cell soma resembled the cyclic variation in the rate of advance of the nucleus seen in migrating fibroblasts (Abercrombie et al., 1970). A likely mechanism for fibroblast migration has been summarized by Abercrombie (1980). In brief, this sequence of
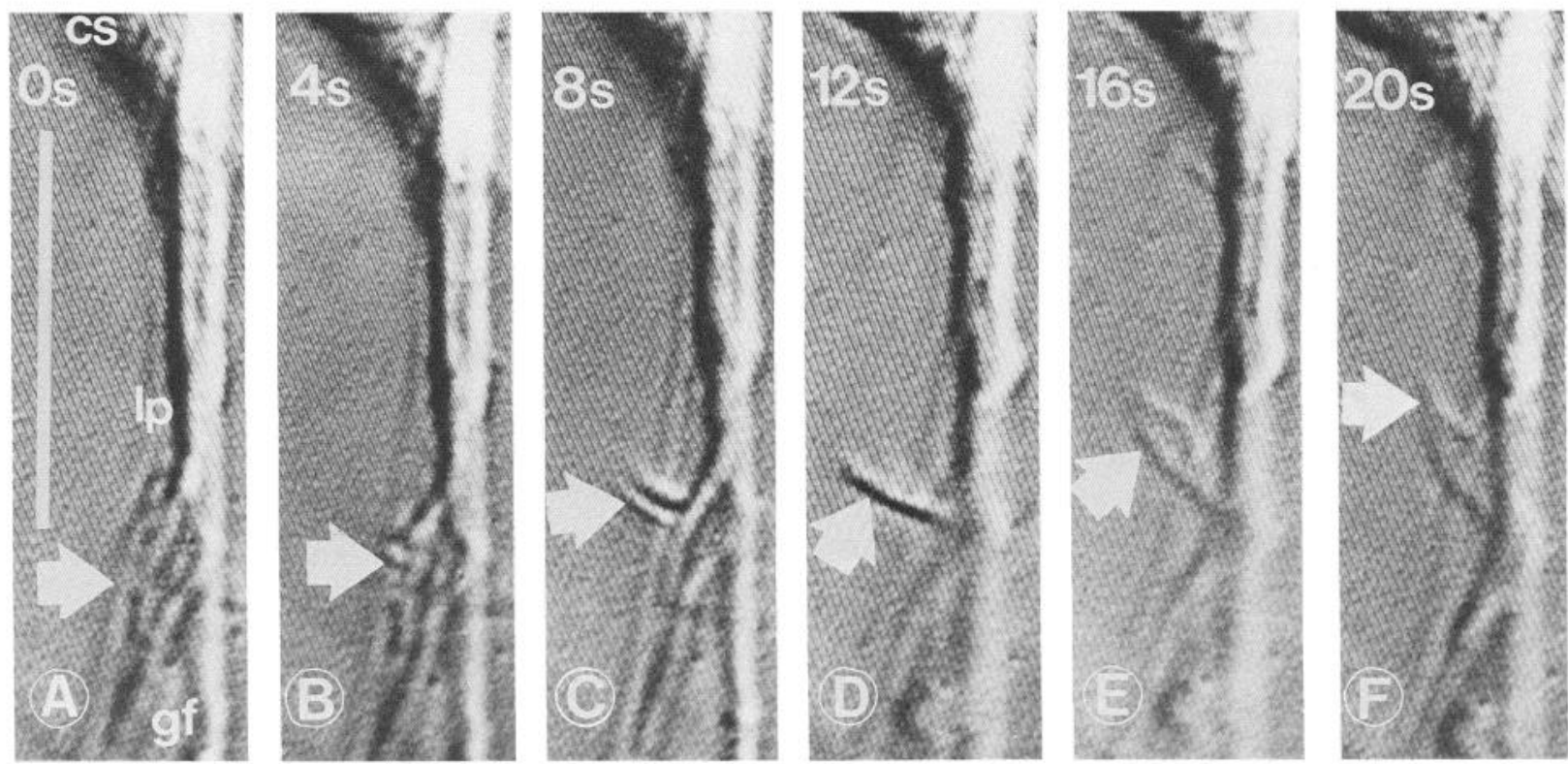

Figure 5. Extension and retraction of a filopodium on the granule neuron leading process. $c s$, Cell soma; $l p$, leading process; arrow, filopodium; $g f$, glial fiber. $A$, The filopodium extends towards the direction of migration. $B-E$, The filopodium rotates backwards towards the cell soma. $F$, The filopodium is resorbed against the leading process. Time interval (in sec) indicated on photographs. Bar, $10 \mu \mathrm{m}$. 

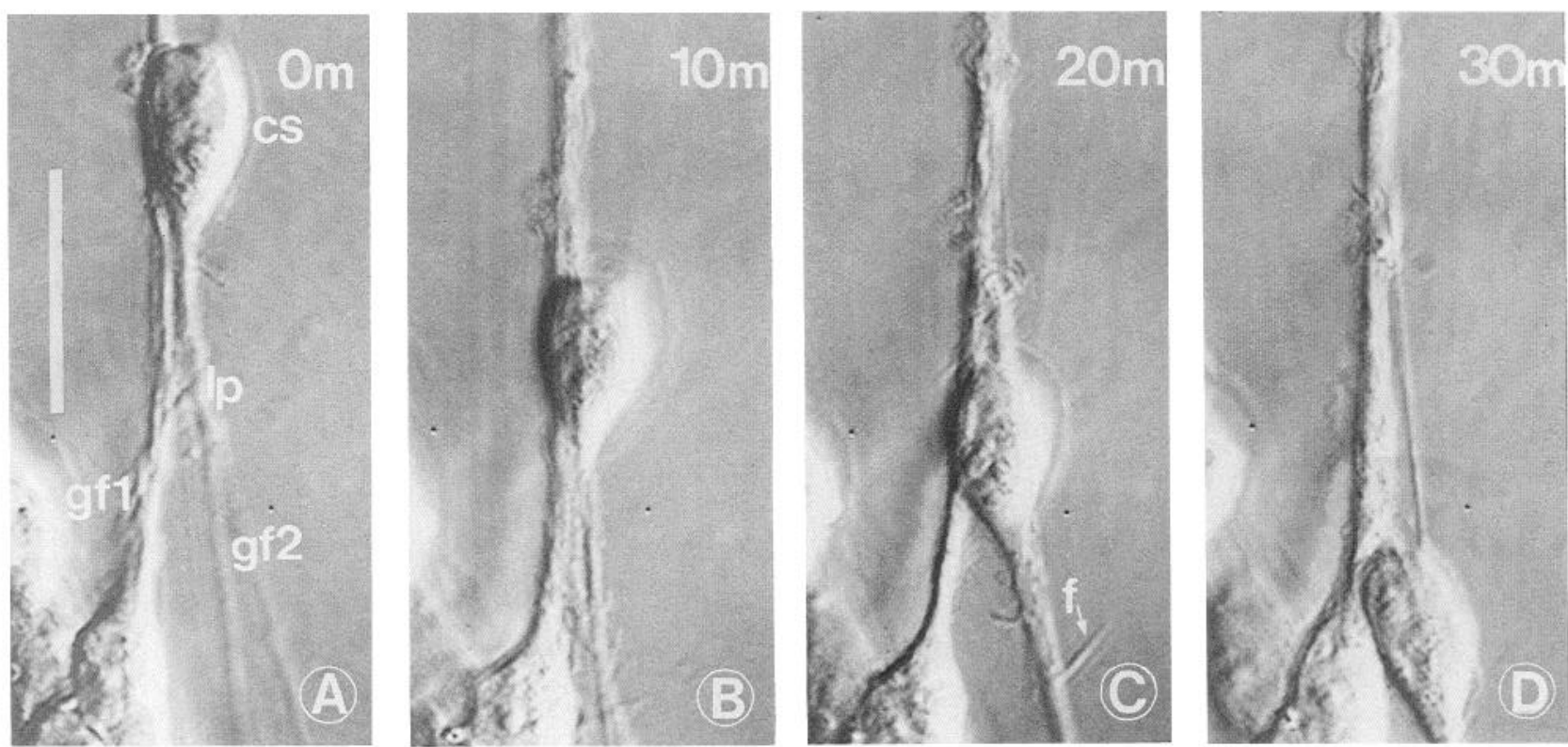

Figure 6. Encounter of a migrating neuron with a second glial fiber. $c s$, Cell soma; $l p$, leading process; $f$, filopodia; $g f 1$, first glial fiber; $g f 2$, second glial fiber. $A$, Neuron approaches intersection of 2 glial fibers; leading process contacts first fiber and extends filopodia to contact second fiber. $B$ and $C$, Granule neuron detaches leading process from first fiber and attaches to second. $D$, Neuron continues migration on second fiber. Time interval (in $\mathrm{min}$ ) indicated on photographs. Bar, $20 \mu \mathrm{m}$.

events for fibroblast migration on flat substrata is as follows: (1) extension of a broad leading lamella, (2) formation within the leading lamella of adhesion sites with the substratum, (3) contraction of intracellular microfilament bundles that connect the nucleus with the cell-substratum adhesion sites, and (4) subsequent movement of the nucleus towards the leading lamella. The issue of how the curvature of the substrate affects the direction of migration has been studied in detail for fibroblasts by Dunn and Heath (1976), who found that fibroblasts could migrate both circumferentially and longitudinally on glass cylinders greater than $200 \mu \mathrm{m}$ in diameter, while they could migrate only in the longitudinal direction on cylinders of smaller diameter. To explain this, Dunn and Heath reasoned from the model for fibroblast migration on flat substrata that the ability to form microfilament bundles parallel to the substrate constrained the direction of migration.

By analogy with these studies, the present time-lapse study suggests that the behavior of migrating granule neurons resem-
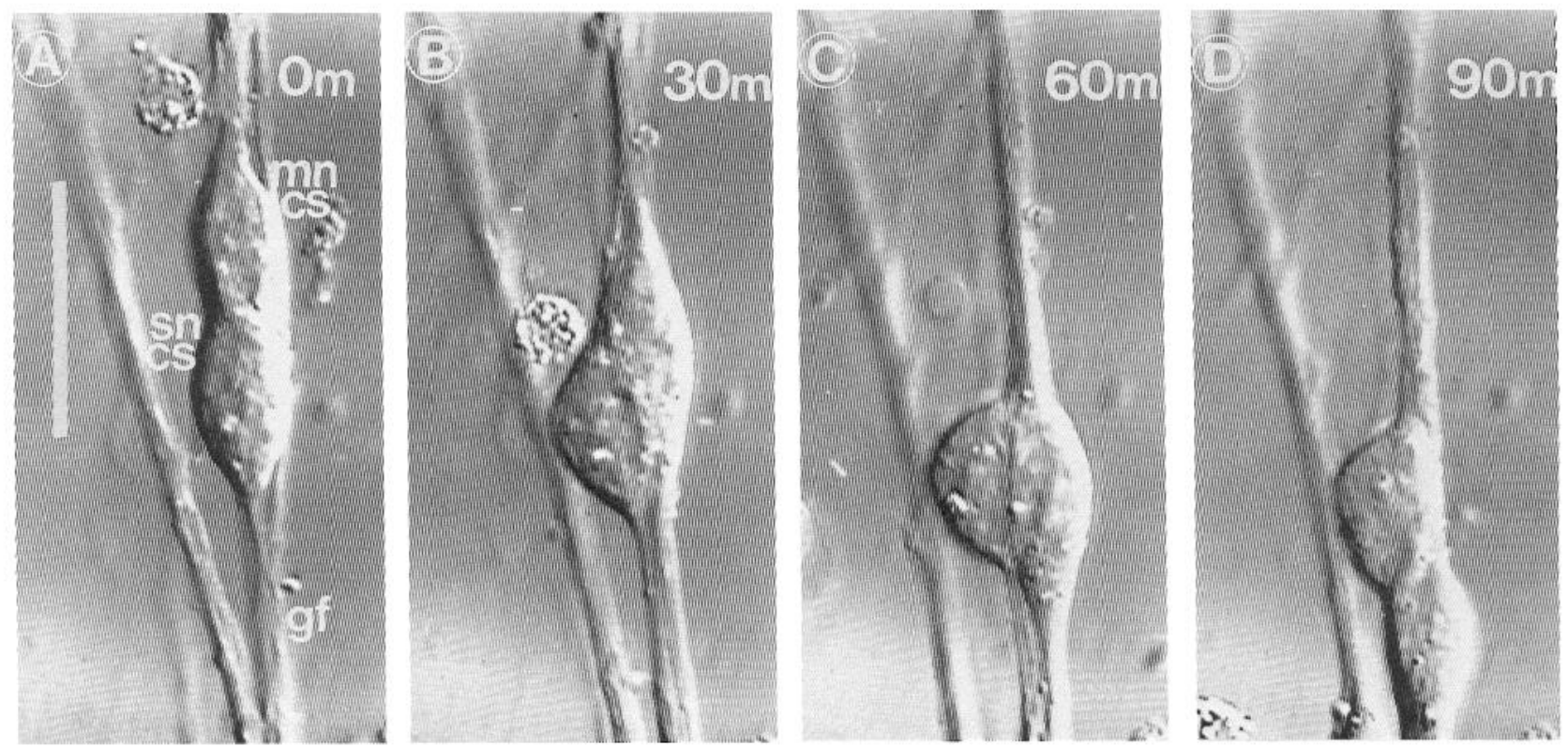

Figure 7. Encounter of a migrating neuron with a stationary neuron on the same glial fiber. mncs, Migrating neuron cell soma; sncs, stationary neuron cell soma; $g f$, glial fiber. Migrating neuron passes between stationary neuron and glial fiber. Note that migration is slowed. Time interval (in min) indicated on photographs. Bar, $20 \mu \mathrm{m}$. 
bles the special case of fibroblast migration on a small, cylindrical substrate. The growing tip of the leading process advanced by rapid extension and retraction of cellular projections, analogous to the rapid advance and retreat of the fibroblast leading lamella, with a net movement in the direction of migration in each case. The more regular cyclic advance and rest of the neuronal cell soma resembled the saltatory movements of the fibroblast nucleus. Consistent with the findings of Dunn and Heath on the orientation of fibroblast migration on small cylinders, granule neurons oriented their migration longitudinally on the Bergmann glial fibers.

Although the present experiments suggest a correlation between granule neuron migration and fibroblast migration, the exact cellular mechanism of granule neuron migration remains unknown. In particular, a role for an actin-myosin-based contractile mechanism within the leading process and the role of cell-surface neuron-glia binding ligands, especially those that might interconnect with cytoskeletal elements, remain to be established. High-resolution video microscopy should provide a useful method for assaying the effects on migrating neurons of pharmacological agents that disrupt cytoskeletal function or of antibodies to neuronal or glial cell-surface components. It will be of interest to examine the effects of Fab fragments specific for astrotactin, a glycoprotein that mediates cerebellar neuronglial interactions in vitro (J. C. Edmondson, R. K. H. Liem, and M. E. Hatten, unpublished observations).

\section{References}

Abercrombie, M. (1980) The crawling movement of metazoan cells. Proc. R. Soc. London [Biol.] 207: 129-147.

Abercrombie, M., J. E. M. Heaysman, and S. M. Pegrum (1970) The locomotion of fibroblasts in culture I. Movements of the leading edge. Exp. Cell Res. 59: 393-398.

Allen, R. D., N. S. Allen, and J. L. Travis (1981) Video-enhanced contrast, differential interference contrast (AVEC-DIC) microscopy: A new method capable of analyzing microtubule-related motility in the reticulopodial network of Allogromia laticollaris. Cell Motil. 1: 291302.

Dunn, G. A., and J. P. Heath (1976) A new hypothesis of contact guidance in tissue cells. Exp. Cell Res. 101:1-14.

Gregory, W. A., J. C. Edmondson, M. E. Hatten, and C. A. Mason (1986) Electron microscopic analysis of video-observed neurons migrating along glia in vitro. Soc. Neurosci. Abstr. 12: 369.

Hatten, M. E. (1985) Neuronal regulation of astroglial morphology and proliferation in vitro. J. Cell Biol. 100: 384-396.

Hatten, M. E., and R. K. H. Liem (1981) Astroglia provide a template for the organization of cerebellar neurons in vitro. J. Cell Biol. 90: $622-630$.

Hatten, M. E., R. K. H. Liem, and C. A. Mason (1984) Two forms of astroglia interact differently with cerebellar neurons in vitro. J. Cell Biol. 98: 193-204.

Hatten, M. E., R. K. H. Liem, and C. A. Mason (1986) Weaver mouse cerebellar granule neurons fail to migrate on wild-type astroglial processes in vitro. J. Neurosci. 6: 2676-2683.

Marsh, L., and P. C. Letourneau (1984) Growth of neurites without filopodial or lamellipodial activity in the presence of cytochalasin B. J. Cell Biol. 99: 2041-2047.

Rakic, P. (1971) Neuron-glia relationship during granule cell migration in developing cerebellar cortex. A Golgi and electronmicroscopic study in Macacus rhesus. J. Comp. Neurol. 141: 283-312.

Rakic, P. (1974) Neurons in the rhesus monkey visual cortex: Systematic relation between time of origin and eventual disposition. Science 183: 425-427.

Rakic, P., and R. L. Sidman (1973) Weaver mutant mouse cerebellum: Defective neuronal migration secondary to abnormality of Bergmann glia. Proc. Natl. Acad. Sci. USA 70: 240-244.

Ramón y Cajal, S. (1911) Histologie du Systeme Nerveux de l'Homme et des Vertebres, Maloine, Paris. [Reprinted by Consejo Superior de Investigaciones Cientificas, Madrid, 1955, Vol. 2.]

Sotelo, C., and P. Changeaux (1974) Bergmann fibers and granule cell migration in the cerebellum of homozygous weaver mutant mouse. Brain Res. 77: 484-491.

Trenkner, E., and R. Sidman (1977) Histogenesis of mouse cerebellum in microwell cultures: Cell reaggregation and migration, fiber and synapse formation. J. Cell Biol. 75: 915-940. 